\title{
Total anomalous pulmonary vein drainage in a 60 -year-old woman diagnosed in an ECG-gated multidetector computed tomography - a case report and review of literature
}

\author{
Elżbieta Czekajska-Chehabb ${ }^{1 A, B, C, D, E, F}$, Andrzej Tomaszewski ${ }^{B, C, D}$, Piotr Adamczyk ${ }^{3 B, C, D}$, \\ Magdalena Zakościelna ${ }^{1 A, B, C, D, E, F}$, Agnieszka Wojtkowska ${ }^{4 B, C, E}$, Elżbieta Siek ${ }^{1 B, C, D}$, \\ Grzegorz Staśkiewicz ${ }^{1 B, C, D}$, Andrzej Drop ${ }^{1 A, D, E}$ \\ ${ }^{1}$ st Department of Medical Radiology, Medical University of Lublin, Lublin, Poland \\ ${ }^{2}$ Department of Cardiology, Medical University of Lublin, Lublin, Poland \\ ${ }^{3}$ Department of Radiology, Public Independent Clinical Hospital Number 4, Lublin, Poland \\ ${ }^{4}$ Department of Cardiology, Public Independent Clinical Hospital Number 4, Lublin, Poland
}

\section{Abstract}

Purpose: Total anomalous pulmonary vein drainage (TAPVD) is a congenital cardiac defect in which there is no connection between pulmonary veins and the left atrium. Pulmonary veins form a confluence independent of the left atrium and drain to a systemic vein. TAPVD types are: supracardiac, cardiac, infracardiac, and mixed. TAPVD accounts for approximately $1.5-2.2 \%$ of all congenital heart diseases. This anomaly is usually diagnosed in the neonatal period, and it coexists with atrial septal defect. Adult cases of TAPVD are rarely reported.

Case report: We report a rare case of a 60-year-old woman with incidentally found, uncorrected TAPVD in ECG-gated multidetector computed tomography. In previous echocardiographic examinations partial anomalous pulmonary venous return and atrial septal defect were diagnosed.

Conclusions: ECG-gated multidetector computed tomography is a valuable diagnostic method for adults with congenital heart disease. It enables evaluation of coronary arteries and simultaneously provides detailed anatomy of great vessels.

Key words: pulmonary veins, congenital heart disease, total anomalous pulmonary venous drainage, multidetector computed tomography.

\section{Introduction}

The incidence of congenital heart disease varies from $0.4 \%$ to $5 \%$ of live births [1]. Partial anomalous pulmonary venous connection (PAPVC) has a prevalence of $0.4-0.7 \%$ [2]. Total anomalous pulmonary venous drainage (TAPVD) accounts for approximately 1.5-2.2\% of all congenital heart diseases and $0.008 \%$ of live births $[3,4]$. This anomaly is usu- ally diagnosed in the neonatal period and coexists with atrial septal defect. Adult patients with TAPVD are rarely seen.

TAPVD is a congenital cardiac disease in which none of the pulmonary veins reaches the left atrium. The pulmonary veins form a confluence that drains to the systemic vein independent of the left atrium [5]. Depending on the anatomic level of the anomalous connection, Darling et al. [6] proposed a classification of TAPVD:

Correspondence address:

Magdalena Zakościelna, Department of Medical Radiology, Medical University of Lublin, 8 Jaczewskiego St., 20-954 Lublin, Poland,

e-mail: magdalenazakoscielna@wp.pl

Authors' contribution:

A Study design · B Data collection · C Statistical analysis · D Data interpretation - E Manuscript preparation · F Literature search · G Funds collection 
- Type I - supracardiac - pulmonary veins drain to the remnant of the left superior vena cava, vertical vein, or the right superior vena cava;

- Type II - intracardiac - pulmonary veins drain to the coronary sinus or right atrium;

- Type III - infracardiac - pulmonary veins drain directly into the inferior vena cava, its tributaries, or the portal venous system;

- Type IV - mixed - pulmonary veins drain into at least two of the above locations.

\section{Case report}

A 60-year-old female $(70 \mathrm{~kg}, 165 \mathrm{~cm})$ was referred to the Department of Cardiology from cardiological health resort for radiofrequency ablation due to recurrent atrial fibrillation. The woman knew that she had congenital heart disease, because she was diagnosed in childhood with atrial septal defect. The patient's mother did not agree to surgical treatment or further diagnostic procedures. The patient complained of recurrent respiratory infections. She was granted incapacity benefit because of inability to perform professional work. She was pregnant once, with miscarriage. She also suffered from depression and nodular goitre.

Numerous echocardiographic examinations were provided, and PAPVC was diagnosed. Left pulmonary vein ostia were described as probably normal. There were also different interpretations of atrial septal defect by different echocardiographers, who suggested ASD type II or ASD sinus venosus.

The patient's ECG showed normal sinus rhythm, left axis deviation, a right bundle branch block and features of right atrial enlargement.

On physical examination, no hepatomegaly, peripheral clubbing, or oedema were observed. Blood test results (morphology and biochemical analysis) were normal.

Transthoracic echocardiography (TTE) performed before catheter radiofrequency ablation demonstrated significantly dilated, hypertrophic right ventricle (RVD1: $62 \mathrm{~mm}, \mathrm{RVD} 2: 74 \mathrm{~mm}, \mathrm{RVD} 3: 85 \mathrm{~mm}$ ), dilatation of pulmonary trunk $(40 \mathrm{~mm})$, "paradoxical" motion of interventricular septum, and atrial septal defect $(30 \mathrm{~mm})$. Pulmonary artery systolic pressure was $103 \mathrm{mmHg}$. Severe tricuspid and moderate pulmonary valve regurgitation were visualised. Left ventricular size and function were normal. A dilated vertical vein in the suprasternal view was detected.

In transoesophageal echocardiographic examination (TEE) conducted before ablation, no echogenic blood or thrombus in heart chambers were found. Cryoablation of cavo-tricuspid isthmus was performed.

ECG-gated, contrast-enhanced 64-row computed tomography was performed to evaluate anomalous pulmonary vein drainage and coronary arteries. The $90-\mathrm{ml}$ iodinated contrast medium iopromide $370 \mathrm{mg}$ was injected intravenously at $4.5 \mathrm{ml} / \mathrm{s}$ followed by $30 \mathrm{ml}$ saline. Images were reconstructed between $5 \%$ and $95 \%$ of the RR interval at $10 \%$ distance. The heart rate was 83 beats per minute.

CT examination revealed dilatation of all pulmonary veins. Right pulmonary veins formed a common trunk (27 mm diameter) situated between descending aorta and right pulmonary artery. Above the left atrial appendage, the trunk joined the left inferior pulmonary veins and formed a vertical vein (47 $\mathrm{mm}$ diameter) (Figure 1).

Left superior pulmonary vein $(33 \times 19 \mathrm{~mm})$ joined the vertical vein above this confluence. Superior vena cava (64 $\mathrm{mm}$ diameter) was formed by the typical right brachiocephalic vein and wide left brachiocephalic vein. All the findings were typical of supracardiac type of TAPVD (Figures 2A-B).

The ASD type II $\left(27 \times 22 \mathrm{~mm}\right.$ dimensions, $409 \mathrm{~mm}^{2}$ area) was also found. There was a left axis deviation of the heart $\left(99.5^{\circ}\right)$. The heart apex was located posteriorly to the left mid axillary line (Figure 3).

Interatrial septum showed maximal deviation of $25 \mathrm{~mm}$ towards the left atrium. The interventricular septum was flattened in systolic phase. Contrast medium reflux into the inferior vena cava was seen. Thickening of mitral and pulmonary valve cusps with mitral valve minor calcifications were noticed. Pulmonary trunk was dilated to $54 \mathrm{~mm}$, right pulmonary artery achieved $38 \mathrm{~mm}$, left pulmonary artery reached $33 \mathrm{~mm}$ (Figures 4A-B).

Cardiac function parameters were as follows:

- The right atrium: EDV 416 ml, ESV 287 ml, SV 129 ml, EF 31\%;

- The right ventricle: EDV 550 ml, ESV 353 ml, SV 197 ml, EF 35\%;

- The left atrium: EDV 103 ml, ESV 46 ml, SV 56 ml, EF 55\%;

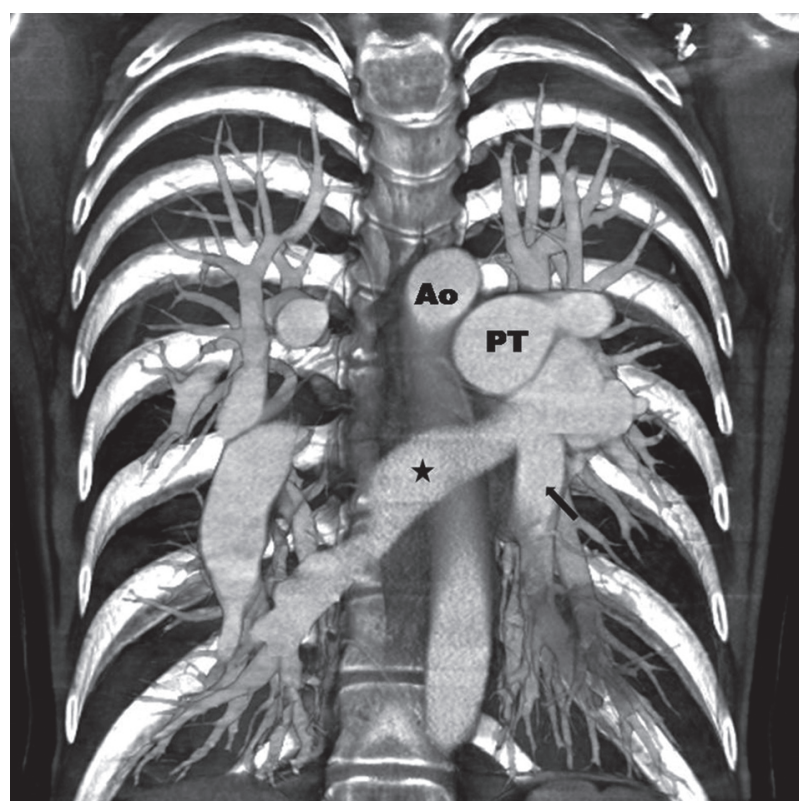

Figure 1. Maximum intensity projection reconstruction of the pulmonary vessel trunk formed by right pulmonary veins (star) and left inferior pulmonary vein (arrow). A0 - aorta, PT - pulmonary trunk 

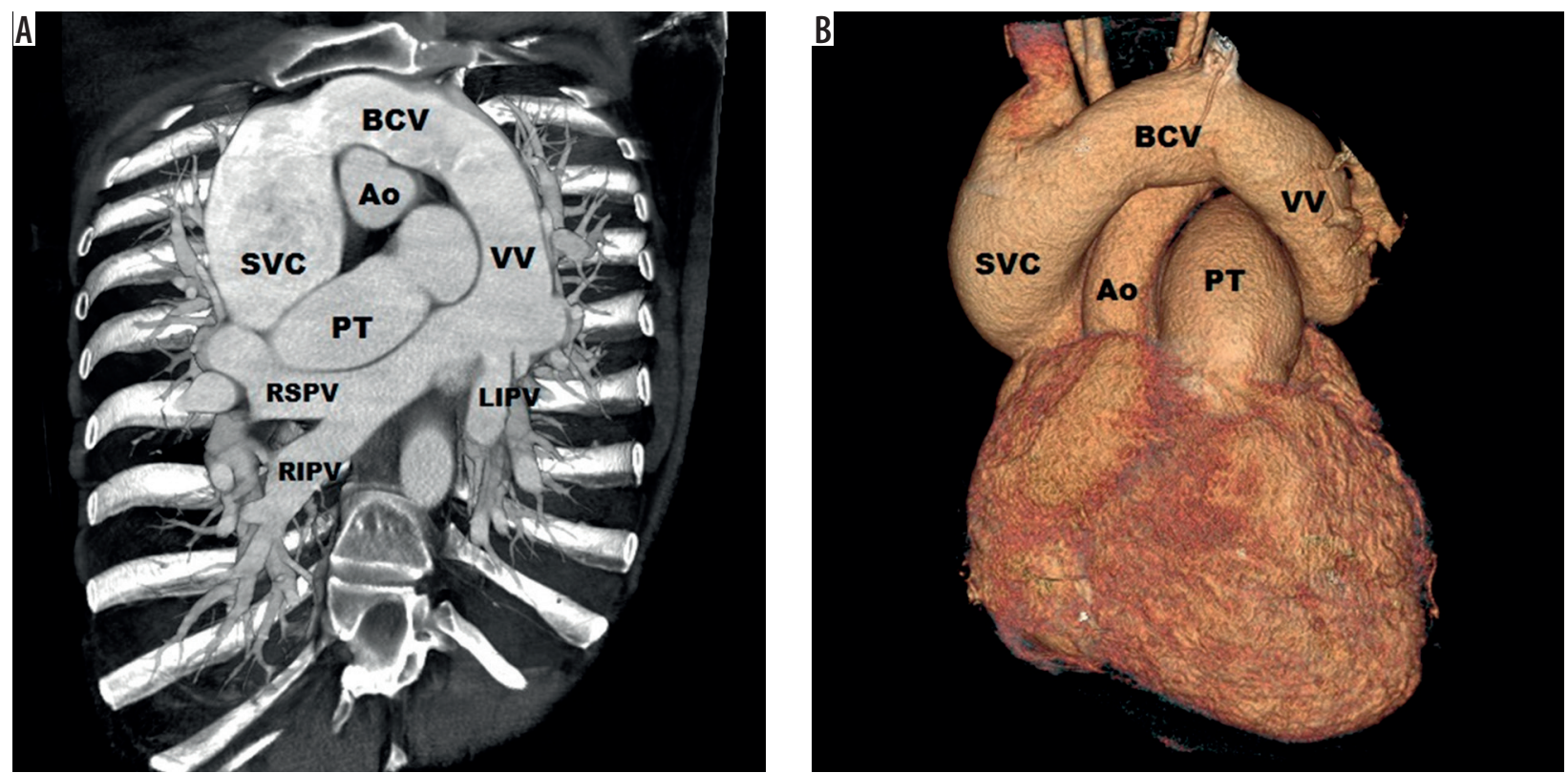

Figure 2. Maximum intensity projection (A) and 3D (B) reconstruction of the vessel arch formed by vertical vein, left brachiocephalic vein, and superior vena cava. RSPV - right superior pulmonary vein, RIPV - right inferior pulmonary vein, LIPV - left inferior pulmonary vein, VV - vertical vein, BCV - brachiocephalic vein, SVC - superior vena cava, Ao - aorta, PT - pulmonary trunk

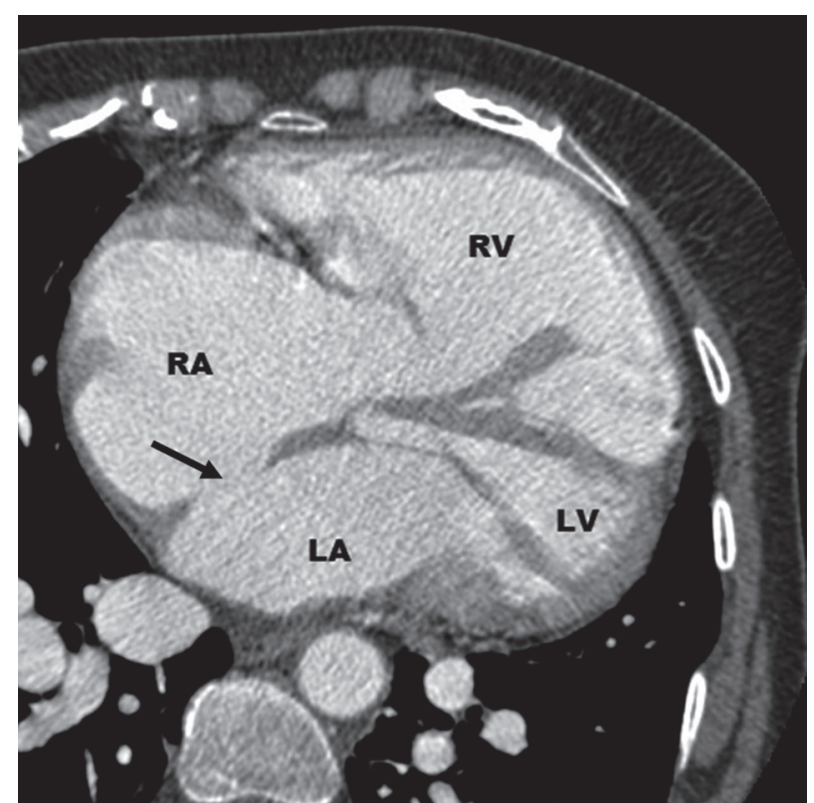

Figure 3. Axial scan, enlargement of right atrium (RA) and right ventricle (RV), ASD type II (arrow), heart apex dislocation to the left. LA - left atrium, LV - left ventricle

\section{- The left ventricle: EDV $120 \mathrm{ml}$, ESV $57 \mathrm{ml}$, SV $63 \mathrm{ml}$,} EF $52 \%$.

No thrombus in the left atrium was found. The left atrial appendage had a 'cauliflower' shape according to the Ernst classification [7]. There were no anomalies or significant stenoses of coronary arteries. Peripheral pulmonary vessels were dilated and mosaic perfusion was noted, which is characteristic for pulmonary hypertension (Figures 5A-B).

The patient was sent for further diagnostics procedures (including right-heart catheterisation) to another medical centre where TAPVD was confirmed and haemodynamic parameters were estimated.

\section{Discussion}

ECG-gated CT is a recognised method of assessment of cardiac and great vessel anatomy prior to ablation treatment, and its complimentary role in echocardiography has been reported [8]. In the majority of patients, ECG-gated $\mathrm{CT}$ is performed to measure the ostia of pulmonary veins and to identify their anatomical variants. Rarely, patients scheduled for ablation present with congenital anomalies, particularly anomalous pulmonary venous drainage. The presented case is the first such finding observed at our institution, among 977 patients. Available literature report only a few cases of anomalous pulmonary venous drainage patients qualified for ablation [9-12].

The presented case shows that even total anomalous pulmonary venous drainage may remain undetected in a patient over 60 years old, with several previous TTEs performed at multiple institutions.

Literature confirms the difficulties in complete assessment of pulmonary veins in TTE [13].

The anomalies observed in our patients may have significantly influenced the scheduled ablation: typically, abnormal impulses originate in the pulmonary vein ostia at the left atrium; then, isolation of pulmonary vein is performed with balloon cryoablation. In the case of anomalous pulmonary venous drainage, patients are treated with high-frequency ablation, with preparation of a detailed electrical potential map of both atria or right ventricle. Agarwal et al. report a case that required SVC isolation in a patient with persistent $\mathrm{AF}$ and anomalous pulmonary vein connection of the right superior pulmonary vein into the SVC [9]. 

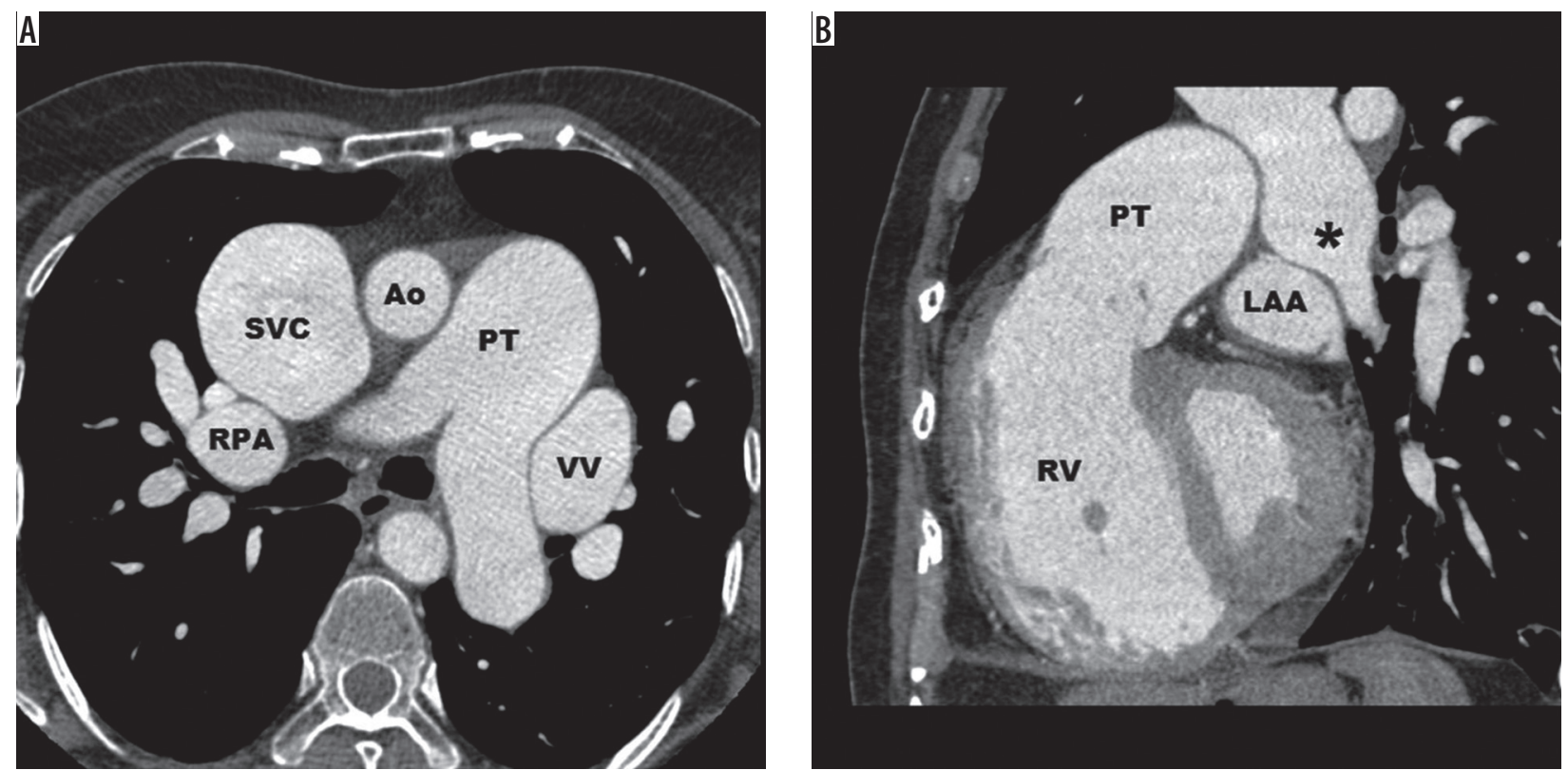

Figure 4. Axial scan (A) dilatation of pulmonary trunk (PT), right pulmonary artery (RPA). SVC - superior vena cava, Ao - aorta, VV - vertical vein. Sagittal reconstruction (B) - enlargement of right ventricle (RV), pulmonary valve cusps thickening, dilatation of pulmonary trunk, right pulmonary vein confluence $\left({ }^{*}\right)$, LAA - left atrial appendage
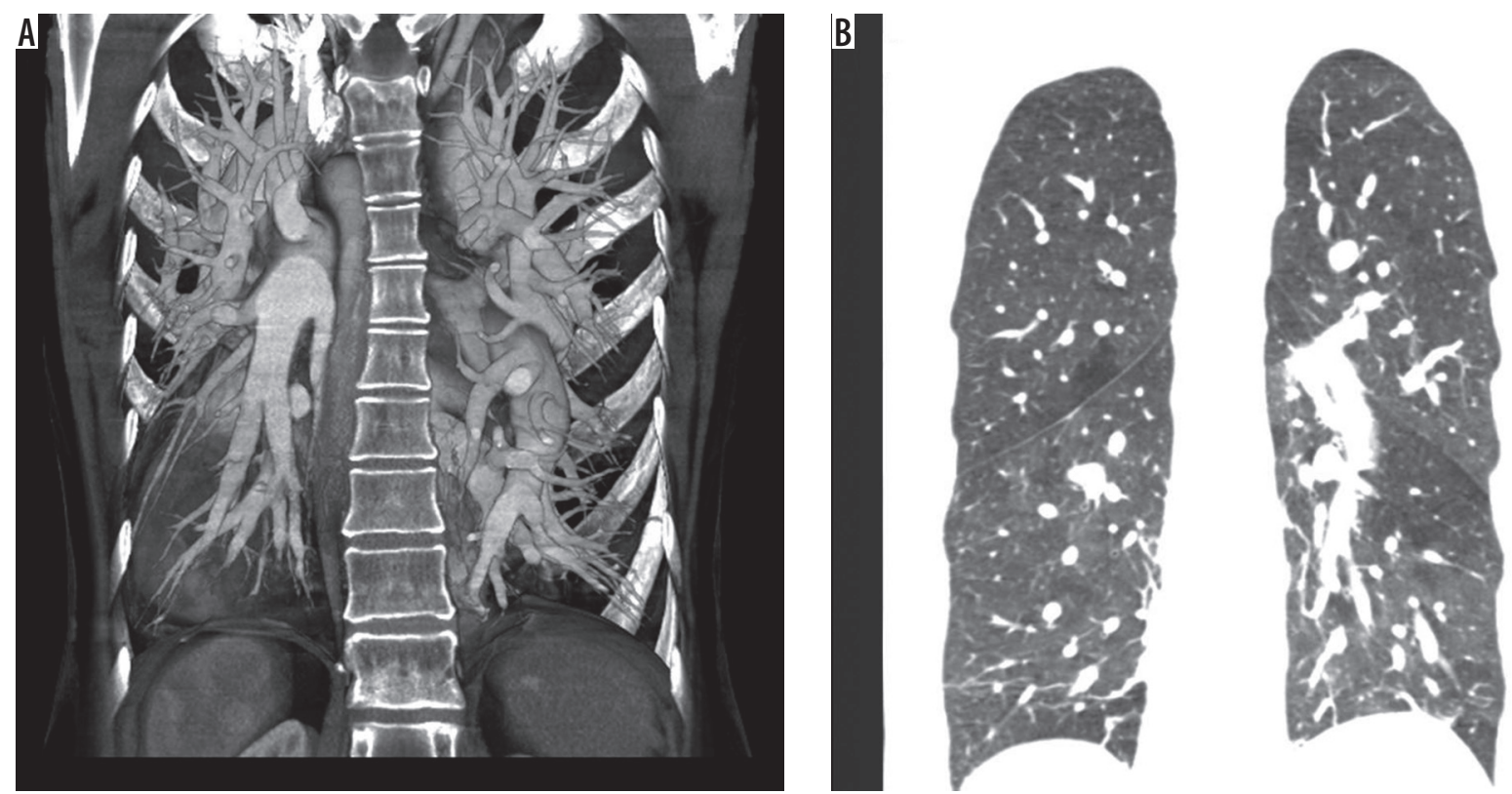

Figure 5. MIP reconstruction (A) of the dilated peripheral pulmonary vessels. Coronal reconstruction (B) mosaic perfusion of the lungs

From clinical perspective, in a patient aged over 60 years, concomitant assessment of coronary arteries has also been important, to exclude significant atherosclerosis.

TAPVD is an uncommon congenital cardiac disease in which there is no connection between pulmonary veins and the left atrium. TAPVD can lead to death in the neonatal period if there is no connection between pulmonary and systemic circulation. ASD allows oxygenated and deoxygenated blood mix. The volume of mixed blood that gets to the systemic circulation depends on the size of the ASD. The size of the leak determinates patients' symptoms. Most patients with TAPVD present cyanosis and congestive heart failure in the early neonatal period, which is why most of them require an emergency surgical intervention [3]. The mortality is estimated at $80 \%$ within the first year of life if surgical repair is not performed [14]. Patients with supracardiac and cardiac type of TAPVD live longer than those with infracardiac and mixed type [15]. The supracardiac type is rarely associated with pulmonary vein obstruction, unlike infracardiac type, in which obstruction reaches $78 \%$ of cases [2].

According to Talwar et al. [16], in a group of 27 patients who underwent surgery for TAPVD at the age of 15-48 years, the most frequent kind of this congenital 
heart disease was supracardiac type $(n=15)$. Other types were: cardiac $(n=7)$ and mixed $(n=5)$. In this group there was no infracardiac type of TAPVD. Angio-CT was performed in one patient. In patients with supracardiac type of TAPVD a wide anastomosis was made between the common chamber and the left atrial appendage by the posterior approach.

Not many patients were diagnosed TAPVD over the age of 40 years [15,17-19]. According to literature data, the oldest patient diagnosed with chest MDCT was 64 years old. To confirm the diagnosis CMR and ECG-gated CT were performed [15].

In the available literature the authors found only one case of TAPVD diagnosed with ECG-gated MDCT in this age group [20]. There are a few cases of ECG-gated MDCT performed to diagnose TAPVD, but in a younger group of patients (below the age of 40 years) $[21,22]$.

Echocardiography is considered to be the first-line modality in the diagnosis of congenital heart defects, including TAPVD, but it is constrained by acoustic windows and limited in its ability to display the complex three-dimensional relationships that are found in some patients with pulmonary venous anomalies [23,24].

Chest CT enables diagnosis or confirmation of TAPVD, but ECG-gated CT provides many additional information of heart structures. ECG-gated MDCT is routinely performed to visualise coronary arteries and cardiac anatomy. This modality has high accuracy for the diagnosis of coronary artery disease, but it also enables non-invasive assessment of patients with suspected congenital heart defects, including assessment of pulmonary veins and left atrium as well as anomalous pulmonary vein drainage [25]. Gating techniques are used to improve temporal resolution and minimise imaging artefacts caused by cardiac motion. It is a fast, easily achievable examination. ECG-gated CT results in high diagnostic image quality. Multi-planar reconstruction (MPR), curved-planar reconstruction (CPR), maximum intensity projection (MIP), and volume render (VR) reconstructions and detailed cardiac function parameters can be obtained. ECG-gated CT correctly depicts all anomalous vascular structures including pulmonary anomalous drainage. Modern computed tomography scanners with 256 or 320 detectors provide higher quality of images, and due to iterative algorithms the patient is exposed to less radiation. Shorter scan time limits the motion artefacts and volume of contrast medium [26]. Compared to MRI, ECG-gated MDCT has the advantage of easy availability, shorter time of acquisition, and the possibility of pulmonary parenchyma and coronary artery assessment; however, radiation dose remains an issue.

\section{Conclusions}

ECG-gated MDCT is a valuable method for diagnosis and classification of TAPVD. Even if the patient has an echocardiographic diagnosis of congenital heart defect, a radiologist assessing coronary arteries in ECG-gated CT should also search for coexisting congenital heart diseases such as anomalous pulmonary drainage. High diagnostic image quality is a result of gating techniques. The use of ECG gating effectively "freezes" cardiac pulsation and vascular wall motion and reduces motion artefacts.

\section{Conflict of interest}

The authors report no conflict of interest.

\section{References}

1. Hoffman JI, Kaplan S. The incidence of congenital heart disease. J Am Coll Cardiol 2002; 39: 1890-1900.

2. Dillman JR, Yarram SG, Hernandez RJ. Imaging of pulmonary venous developmental anomalies. AJR Am J Roentgenol 2009; 192: 1272-1285.

3. Ashfaq A, Shah N, Khan MZ, et al. Successful surgical intervention of total anomalous pulmonary venous drainage in the third decade of life. Ann Med Surg (Lond) 2013; 2: 60-62.

4. Nabati M, Bagheri B, Habibi V. Coincidence of total anomalous pulmonary venous drainage to the superior vena cava, common atrium, and single ventricle: a very rare condition. Echocardiography 2013; 30: E98-101.

5. Oliver JJ, Michael HC, English KM, et al. Unusual Causes of Late Right Heart Dilatation Long After Repair of Total Anomalous Pulmonary Venous Drainage. Circulation 2013; 127: 258-259.

6. Craig JM, Darling RC, Rothney WB. Total pulmonary venous drainage into the right side of the heart; report of 17 autopsied cases not associated with other major cardiovascular anomalies. Lab Invest 1957; 6: 44-64.
7. Beigel R, Wunderlich NC, Ho SY, et al. The left atrial appendage: anatomy, function, and noninvasive evaluation. JACC Cardiovasc Imaging 2014; 7: 1251-1265.

8. Burke SJ, Aggarwala G, Stanford W, et al. Preablation assessment for the left atrium: comparison of ECG-gated cardiac CT with echocardiography. Acad Radiol 2008; 15: 835-843.

9. Agarwal SC, Bittinger L, Tang AS. Importance of superior vena cava isolation in successful ablation of persistent atrial fibrillation in patient with partial anomalous pulmonary vein. Pacing Clin Electrophysiol 2013; 36: e146-149.

10. Schreiber D, Müller K, Kottkamp H. Catheter ablation in a patient with paroxysmal atrial fibrillation and partial anomalous pulmonary vein connection. J Cardiovasc Electrophysiol 2011; 22: 717.

11. Fukamizu S, Sakurada H, Nishizaki M, Hiraoka M. Catheter ablation in a patient with partial anomalous pulmonary venous return and atrial fibrillation. Heart Rhythm 2012; 9: 1002-1003.

12. Huang H, Yang B, Jiang H, et al. Catheter ablation of paroxysmal atrial fibrillation in a young patient with a partial anomalous 
pulmonary venous connection. Chin Med J (Engl) 2010; 123: 2284-2286

13. Braksator W, Kuch M, Difenbach C, et al. Two-dimensional transesophageal echocardiography (clinical value). Kardiol Pol 1992; 37: 383-388.

14. Gathman GE, Nadas AS. Total anomalous pulmonary venous connection: clinical and physiologic observations of 75 pediatric patients. Circulation 1970; 42: 143-154.

15. Ogawa $M$, Nakagawa $M$, Hara $M$, et al. Total anomalous pulmonary venous connection in a 64-year-old man: a case report. Ann Thorac Cardiovasc Surg 2013; 19: 46-48.

16. Talwar S, Choudhary SK, Reddy S, et al. Total anomalous pulmonary venous drainage beyond childhood. Interact Cardiovasc Thorac Surg 2008; 7: 1058-1061.

17. Nurkalem Z, Gorgulu S, Eren M, et al. Total anomalous pulmonary venous return in the fourth decade. Int J Cardiol 2006; 113: 124-126.

18. Feng Q, Wu S, Yu G. Surgical treatment of a 56-yearold woman with an intracardiac type of total anomalous pulmonary venous connection. Thorac Cardiovasc Surg 2010; 58: 175-176.

19. Kong D, Pan C, Dong L, et al. Rare variant of mixed total anomalous pulmonary venous drainage (TAPVD) in a 55-year-old patient. Eur Heart J Cardiovasc Imaging 2015; 16: 227.

20. Funabashi N, Takaoka H, Irie R, et al. Supra-cardiac type (1a) total anomalous pulmonary venous return with right to left shunt atrial septal defect diagnosed by 320 slice CT. Eur Heart J 2015; 36: 1776.
21. Kantarci M, Yuce I, Yalcin A, et al. Evaluating adult cor triatriatum with total anomalous pulmonary venous connections by multidetector computer tomography angiography. Folia Morphol 2011; 70: 312-314.

22. Singh N, Singh R, Aga P, et al. Cardiac type of total anomalous pulmonary venous connection: diagnosis and demonstration by multidetector CT angiography. BMJ Case Rep 2013; doi:10.1136/ bcr-2012-007994.

23. Oh KH, Choo KS, Lim SJ, et al. Multidetector CT evaluation of total anomalous pulmonary venous connections: comparison with echocardiography. Pediatr Radiol 2009; 39: 950-954.

24. Dyer KT, Hlavacek AM, Meinel FG, et al. Imaging in congenital pulmonary vein anomalies: the role of computed tomography. Pediatr Radiol 2014; 44: 1158-1168.

25. Ratajczak P, Sławińska A, Martynowska-Rymer I. Anatomical Evaluation of the Pulmonary Veins and the Left Atrium Using Computed Tomography Before Catheter Ablation: Reproducibility of Measurements. Pol J Radiol 2016; 81: 228-232.

26. Restrepo MS, Cerqua A, Turek JW. Pentalogy of Cantrell with ectopia cordis totalis, total anomalous pulmonary venous connection, and tetralogy of Fallot: a case report and review of the literature. Congenit Heart Dis 2014; 9: E129-134. 